\title{
Clinicopathological Characteristics and Survival Rate of Patients with Laryngeal Squamous Cell Carcinoma: A Retrospective Study during 2011-2017 in Yazd, Iran
}

\author{
Shokouh T Zahir ${ }^{1}$, Koorosh Rahmani ${ }^{2}$, Meisam Mehri ${ }^{3}$, Mohammad Shafiee ${ }^{4}$, Seyed M Reza Mortazavizadeh ${ }^{5}$
}

\begin{abstract}
Background: The aim of the current study is to determine the clinicopathological characteristics of patients with laryngeal squamous cell carcinoma and evaluation of the effect of prognostic factors on survival rate.

Materials and methods: The archives of all patients with laryngeal squamous cell carcinoma (2011-2017) were studied. Patients' data were extracted using a checklist that included age, gender, tumor stage, treatment methods used, and survival rate in months. All data were analyzed by the SPSS-17 software with the Chi-square test, the Kaplan-Meier test, and the log-rank test at a statistical significance level of $p<0.05$.

Results: Out of 109 patients with laryngeal cancer, 99 were males and 10 were females. Patients in stages I and II and also in stage III had a longer overall survival compared to patients in stage IV. The mean survival rate was found to be significantly lower in patients in stage IV compared to those in the other stages of the disease. Patients in the age group of 35-59 years had a longer overall survival compared to the older age group (60-89 years). Patients at stage III of the disease who had not undergone chemotherapy had a greater longevity compared to the ones who had undergone the therapy. Patients at stage IV who had not undergone laryngectomy had a much lower survival rate compared to those who had used both surgery and radiotherapy or chemoradiation.

Conclusion: Surgery and radiotherapy can positively affect the mean survival rate in patients at stages I and II of laryngeal carcinoma. Together surgery and chemotherapy or chemoradiation have a positive effect on the survival of patients in advanced stages of the disease.

Keywords: Chemotherapy, Larynx, Radiotherapy, Squamous cell carcinoma.

International Journal of Phonosurgery and Laryngology (2018): 10.5005/jp-journals-10023-1162
\end{abstract}

\section{INTRODUCTION}

Speech and voice are humans' means of communication with the environment around them and with other humans., ${ }^{1,2}$ Malignant laryngeal tumors can be life-threatening in addition to causing speech disorders and the loss of voice. Laryngeal cancer comprises about $2.2 \%$ of all the cases of cancer in males and about $0.4 \%$ of all the cases of cancer in females. ${ }^{3,4}$ Squamous cell carcinoma is the most common type of laryngeal cancer. Approximately 3.2 new cases of laryngeal cancer are reported per 1,00,000 men and women, with an annual mortality rate of about 1.1 per $1,00,000$ men and women. ${ }^{3-6}$ Most patients are in their fifth or sixth decades of life at the time of diagnosis but this cancer has also been reported in younger age groups. Men make up about $96 \%$ of the cases of laryngeal cancer. ${ }^{5-7}$ In Iran, about 90,000 people are affected by different types of cancer each year, and the 11 million cases of cancer reported in 2005 are anticipated to reach 18 million by $2020{ }^{8}$ Nevertheless, according to the statistics provided by the Cancer Research Center of Iran, laryngeal cancer is not among the 10 most common types of cancer in the country., 90 Several risk factors have been reported for laryngeal cancer, including smoking cigarettes and the hookah and the excessive consumption of alcohol. ${ }^{10-12}$ Several factors affect the prognosis of laryngeal cancer, including the stage; site; size; grade of the tumor; the patient's age, gender, and general health status. ${ }^{6-8}$ Treatment may be based on the stage of the disease, the site affected by the tumor, the size of the tumor, the patient's attempt at preserving his speech, eating habits, recurrence of the tumor, and the state of breathing. ${ }^{10-12}$ The type of treatment may vary based on the affected site and the tumor stage and may involve radiotherapy, surgery, and chemotherapy either alone or in combination. ${ }^{1-10}$ The most popular method of treatment

\footnotetext{
1,2Department of Clinical and Surgical Pathology, Shahid Sadoughi University of Medical Sciences, Yazd, Iran

${ }^{3,4}$ Department of Pathology, Shahid Sadoughi University of Medical Sciences, Yazd, Iran

${ }^{5}$ Department of Oncology, Hematology, Azad Medical University, Yazd, Iran
}

Corresponding Author: Mohammad Shafiee, Department of Pathology, Shahid Sadoughi University of Medical Sciences, Yazd, Iran, Phone: +98 9370075901, e-mail: m.shafii.70@gmail.com

How to cite this article: Zahir ST, Rahmani K, et al. Clinicopathological Characteristics and Survival Rate of Patients with Laryngeal Squamous Cell Carcinoma: A Retrospective Study during 2011-2017 in Yazd, Iran. Int J Phonosurg Laryngol 2018;8(2):65-70.

Source of support: Nil

Conflict of interest: None

used for these patients includes partial or total laryngectomy. In the early stages of the disease (stages I and II), radiotherapy or local surgery is considered the first steps in treatment. ${ }^{5-10}$ In the more advanced stages (stages III and IV), the treatment options considered include chemoradiation or total laryngectomy combined with radiation or chemotherapy. ${ }^{11,14,15}$ Each of these treatment options has their own advantages and disadvantages (e.g., complications). ${ }^{14}$ There are different reports on patients' survival rate based on their chosen method of treatment. ${ }^{5-18}$ Given the lack of comprehensive studies conducted on this type of cancer in Iran, the present study aims to investigate cases of laryngeal cancer and the factors affecting the prognosis of this cancer over a 5-year period. 


\section{Materials and Methods}

This descriptive analytical survival study was conducted on all registered patients with primary laryngeal squamous cell carcinoma in Shahid Sadoughi and Shahid Rahnemon educational General Hospitals (two referral centers for Shahid Sadoughi University of Medical Sciences and Health Services in Yazd, Iran) during 2011-2017. The study protocol was reviewed and approved by the medical ethics committee at Shahid Sadoughi University. The samples were selected through the census method. The inclusion criteria were positive laryngeal biopsy or laryngectomy specimens. Patients who had terminated their treatment (for whatever reason) or whose records were not available or whose malignancy involved cases other than squamous cell carcinoma were excluded from the study. All patients are asked to sign an informed consent form upon their freewill agreeing that their medical records be used for research purposes. Patients' demographic information such as age at diagnosis, gender, history of smoking or otherwise, type of lesion, month and year diagnosed with laryngeal cancer, type of cancer and tumor stage, treatment protocols, and telephone number were extracted from the available data. The researchers then visited oncologists and Shahid Ramezanzadeh Radiotherapy Center to identify the type of treatments used for the malignant cases, including surgery, radiotherapy, laryngectomy, and chemotherapy. The data collected were entered into a predesigned checklist. The tumors were divided into three groups based on the anatomical site they involved: group I (supraglottis), group II (glottis), and group III (subglottis). As for the staging of the disease, patients were divided into four stages according to the American Joint Committee on Cancer staging of laryngeal cancer (AJCC) and (TNM), so that stages I and II were considered the early stages and stages III and IV were considered the advanced stages. Tumors were eventually divided into three main groups, including group I (stages I and II), group II (stage III), and group III (stage IV). Following the pathological evaluation, patients were contacted via telephone to acquire information regarding their current status (free of tumor). In case of death, condolences were expressed to patients' family members. The follow-up time was as the interval between the diagnosis time and the time of death or last contact with the patient. Survival was defined as the patient being alive upon the telephone contact.

\section{Statistical Analysis}

Statistical analyses were performed using the SPSS software version 17 for windows (IBM Inc., NY, US). Categorical variables are demonstrated as proportions and continuous variables as mean and standard deviation (SD). In all analyses, continuous variables with non-normal distribution are reported using the median (interquartile range). The Kaplan-Meier method was used to assess the impact of different categorical predictors on patients' survival. Differences in distribution of events between classes of categorical variables are evaluated using the log-rank test.

\section{Results}

Totally, 109 patients were included in the study, 99 (90.8\%) males and $10(9.2 \%)$ females (male/female ratio $=9.9$ ).

Younger patients in the age group of 35-59 years had a longer overall survival (HR: $0.535, \mathrm{Cl} 95 \%, 0.293-0.977, \mathrm{PV}=0.02)$ compared to the older age group (60-89 years).

The mean duration of survival in each stage irrespective of the prognostic factors was $64 \pm 5$ months in stages I and II, $65 \pm 6$ months in stage III, and $38 \pm 5$ months in stage IV, with significant differences between the rates obtained for the different stages (log-rank test, PV =0.007). Patients in stages I and II (HR: $0.343, \mathrm{Cl}$ $95 \%, 0.171-0.690, \mathrm{PV}=0.003$ ) and also in stage III (HR: 0.324, Cl 95\%, $0.145-0.723, \mathrm{PV}=0.006$ ) had a longer overall survival compared to patients in stage IV (Fig. 1A).

Survival of patients based on the location of the tumor showed that patients with subglottic tumors had the lowest survival rate; however, the difference was not statistically significant between groups (log-rank test, $p=0.25$ ) (Fig. 1B).

Survival of patients based on stage of tumor and age groups is given in Table 1. Patients in stages I and II had a significantly lower survival rate in the 60-89 age group compared to the 35-59 age group, comprising a statistically significant difference (log-rank test, $p=0.02$ ). In stage III, however, no significant differences were observed between the two age groups, and the mean survival rate was not affected by age in patients in this stage of the disease (log-rank test, $p=0.81$ ). In stage IV, no significant differences were observed between the two age groups in the mean survival rate (log-rank test, $p=0.51$ ) (Fig. 1C1 to 3 ).

No significant relationship was observed between the mean duration of survival and the stage of the disease or gender (Fig. 1D1 to 3).

No significant differences were observed between the groups in their mean survival rate by the location of tumor and the stage of the disease in either of the stages, including stages I and II (logrank test, $p=0.73$ ), stage III (log-rank test, $p=0.31$ ), and stage IV (log-rank test, $p=0.87$ ) (Fig. 2A1 to 3).

Survival rate based on the stage of tumor and chemotherapy/ radiotherapy is given in Table 2 .

Survival rate based on the stage of tumor and treatment type is given in Table 3. Moreover, no significant differences were observed in the mean survival rate between the patients who had undergone laryngectomy and those who had not undergone the procedure, regardless of whether their laryngectomy was partial or total, and in either of the stages I, II, III, or IV. In stages I and II (log-rank test, $p=0.18$ ) and III (log-rank test, $p=0.09$ ), no significant differences were observed between the groups in their mean survival rate by the type of therapy; however, the difference between the groups was statistically significant in stage IV, so that the mean survival rate was significantly lower in patients who had not undergone laryngectomy, but much higher in patients who had undergone a combination of laryngectomy, chemotherapy, and radiotherapy or a combination of laryngectomy and radiotherapy (log-rank test, $p=0.03$ ) (Fig. 2B1 to 3).

\section{Discussion}

Previous studies have shown a significantly higher frequency of laryngeal cancer in men than in women with an approximate ratio of $7: 1,^{5-13}$ while the present study found this ratio to be slightly higher at (9:1). Laryngeal cancer tends to mostly affect the age group over 50 , and the present study also reported the mean age of the patients as 60 , which was not inconsistent with the findings reported in various other countries. ${ }^{3-13}$ Smoking cigarettes is considered the main etiologic factor in laryngeal cancer. ${ }^{1-3}$ In the present study, $87.2 \%$ of the patients were smokers. In stages I and II, the patients' survival rate was higher in the younger age group compared with the older age group, which is almost consistent with the findings reported by the American Cancer Society, and this finding was also true in patients in stages III and IV of the disease; however, the difference was not statistically significant in the latter stages. ${ }^{6,7}$ 
Survival function

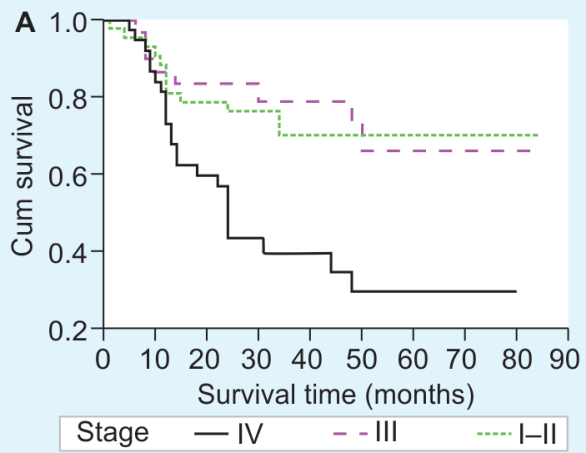

Survival function

C1 Stage: I-II

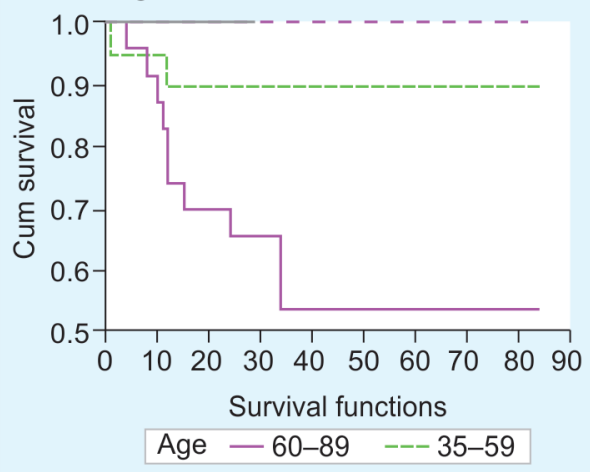

Survival function

D1 Stage: I-II

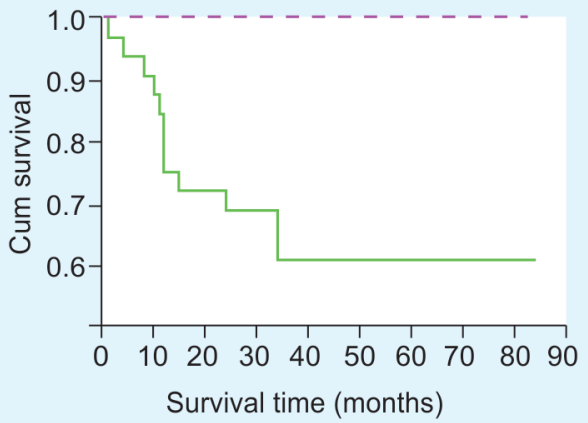

Gender - - Female --- Male

\section{Survival function}

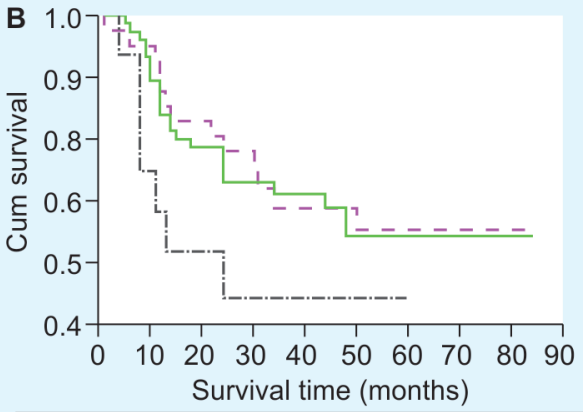

Location - SubGlott - - SupraGlott ----. Glott

Survival function

C2 Stage: III

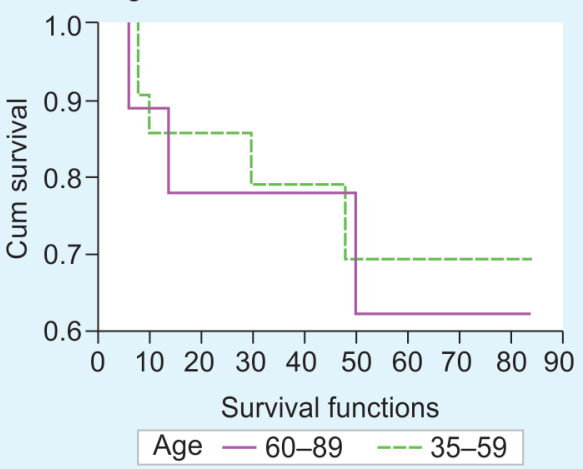

Survival function

D2 Stage: III

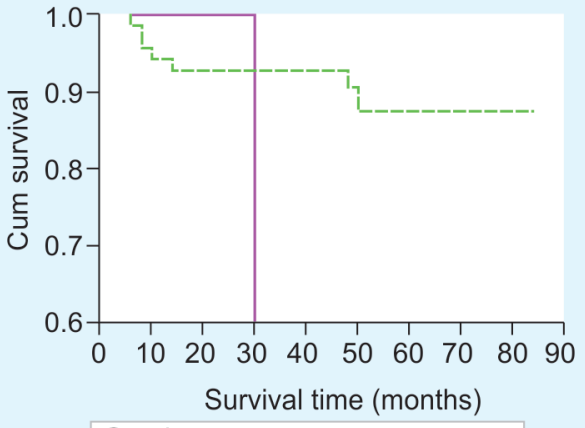

Gender - Female --- Male
Survival function

C3 Stage: IV

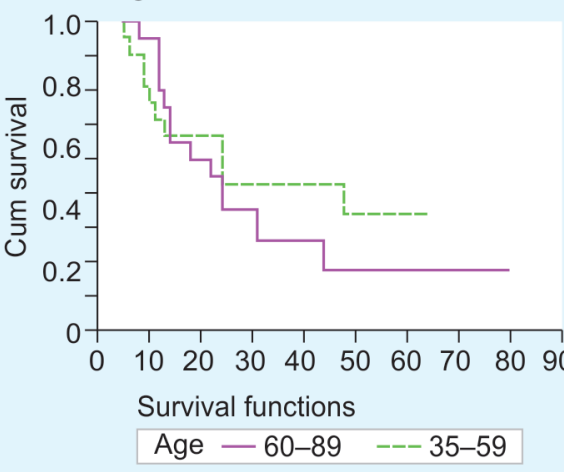

Survival function

D3 Stage: IV

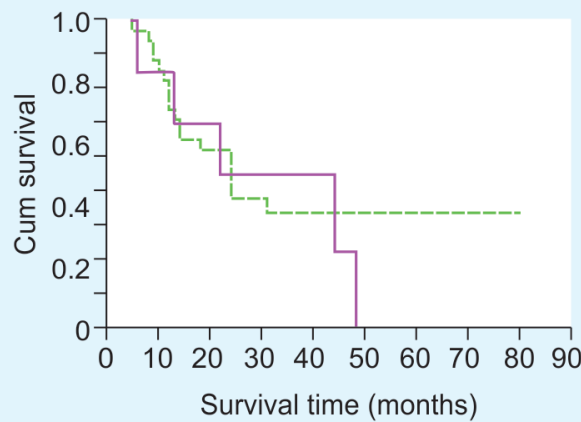

Gender — Female --- Male

Figs 1A to D: (A) Kaplan-Meier survival estimates for laryngeal carcinoma in patients stratified by different stages (I-IV); (B) Kaplan-Meier survival estimates for laryngeal carcinoma in patients stratified by location of tumor; (C1 to 3) Kaplan-Meier survival estimates for laryngeal carcinoma in patients stratified by different stages (I-IV) and patients' age; (D1 to 3) Kaplan-Meier survival estimates for laryngeal carcinoma in patients stratified by different stages (I-IV) and patients' gender

Table 1: Survival of patients based on stage of tumor and age groups

\begin{tabular}{llllccc}
\hline Stage & Age group & $\begin{array}{l}\text { Mean }+S E^{*} \\
\text { (month) }\end{array}$ & Cl95\% & Death $(\mathrm{N})$ & Censored (\%) & $p$ value \\
\hline I-II & $35-59$ & $76+5$ & $65-87$ & 2 & 89.47 & 0.0235 \\
& $60-89$ & $53+7$ & $39-67$ & 10 & 56.52 & 76.19 \\
III & $35-59$ & $66+7$ & $53-80$ & 5 & 66.67 & 0.819 \\
& $60-89$ & $62+10$ & $42-83$ & 3 & 42.11 & 0.511 \\
IV & $35-59$ & $36+6$ & $25-47$ & 11 & 27.78 & \\
& $60-89$ & $32+7$ & $20-45$ & 13 & & \\
\end{tabular}

\footnotetext{
*SE is the standard error
} 


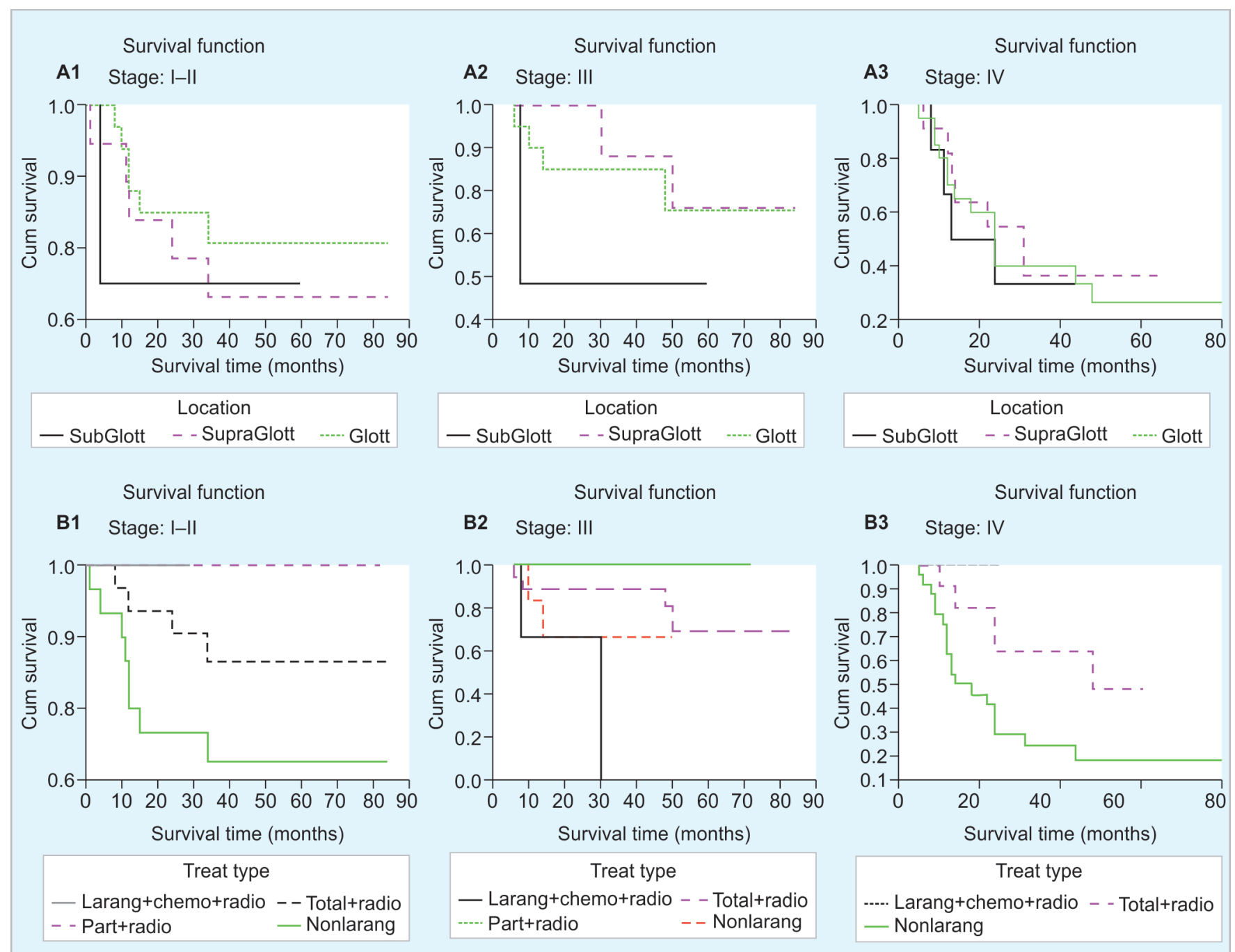

Figs 2A and B: (A1 to 3) Kaplan-Meier overall survival and survival estimates for laryngeal carcinoma in patients stratified by different stages (I-IV) and tumor location; (B1 to 3) Kaplan-Meier survival estimates for laryngeal carcinoma in patients stratified by different stages (I-IV) and treatment types

Table 2: Survival rate based on stage of tumor and chemotherapy/radiotherapy

\begin{tabular}{|c|c|c|c|c|c|c|}
\hline Stage & Chemotherapy & Mean + SE (month) & $\mathrm{Cl} 95 \%$ & Death $(N)$ & Censored (\%) & $p$ value \\
\hline \multirow[t]{2}{*}{ I-II } & No & $68+5$ & $58-79$ & 7 & 78.13 & 0.076 \\
\hline & Yes & $46+11$ & $24-68$ & 5 & 50 & \\
\hline \multirow[t]{2}{*}{ III } & No & $70+6$ & $59-81$ & 5 & 80 & 0.011 \\
\hline & Yes & $22+5$ & $12-33$ & 3 & 40 & \\
\hline \multirow[t]{2}{*}{ IV } & No & $43+8$ & $28-58$ & 10 & 41.18 & 0.325 \\
\hline & Yes & $29+5$ & $19-39$ & 14 & 30 & \\
\hline Stage & Radiotherapy & Mean + SE (month) & $\mathrm{Cl} 95 \%$ & Death (N) & Censored (\%) & $p$ value \\
\hline \multirow[t]{2}{*}{$|-| \mid$} & No & $36+10$ & $16-57$ & 3 & 57.14 & 0.175 \\
\hline & Yes & $66+5$ & $56-76$ & 9 & 74.29 & \\
\hline \multirow[t]{2}{*}{ III } & No & $40+9$ & $23-57$ & 1 & 75 & 0.9556 \\
\hline & Yes & $65+6$ & $53-77$ & 7 & 73.08 & \\
\hline \multirow[t]{2}{*}{ IV } & No & $27+9$ & $9-46$ & 7 & 22.22 & 0.143 \\
\hline & Yes & $36+4$ & $27-44$ & 17 & 39.29 & \\
\hline
\end{tabular}

Previous studies and the American Cancer Society have reported the male to female ratio of mortality in all races and ethnicities to be 1.9:0.4. ${ }^{6,7}$ In the present study, however, the mean survival rate was lower in women compared with that in men in all the stages of the disease; nevertheless, the disparity of findings might have been due to the small female sample size assessed in the present study. ${ }^{6,711}$ Laryngeal tumors occur on three anatomic sites, and different survival rates and prognoses were reported for them 
Table 3: Survival rate based on stage of tumor and treatment type

\begin{tabular}{|c|c|c|c|c|c|c|}
\hline Stage & Treatment type & Mean + SE (month) & $\mathrm{Cl} 95 \%$ & Death $(N)$ & Censored (\%) & $p$ value \\
\hline \multirow[t]{3}{*}{$|-| \mid$} & Non-laryngectomy & $52+9$ & $35-68$ & 8 & 55.56 & 0.187 \\
\hline & Partial laryngectomy + radiotherapy & - & - & 0 & 100 & \\
\hline & Total laryngectomy + radiotherapy & $70+6$ & $57-82$ & 4 & 78.95 & \\
\hline \multirow[t]{4}{*}{ III } & Non-laryngectomy & $37+7$ & $23-52$ & 2 & 66.67 & 0.094 \\
\hline & Partial laryngectomy + radiotherapy & - & - & 0 & 100 & \\
\hline & Total laryngectomy + radiotherapy & $69+7$ & $55-82$ & 4 & 77.78 & \\
\hline & Laryngectomy + chemo + radio & $23+8$ & $6-39$ & 2 & 33.33 & \\
\hline \multirow[t]{3}{*}{ IV } & Non-laryngectomy & $29+5$ & $18-39$ & 19 & 20.83 & 0.037 \\
\hline & Total laryngectomy + radiotherapy & $43+6$ & $31-55$ & 5 & 54.55 & \\
\hline & Laryngectomy + chemo + radio & - & & 0 & 100 & \\
\hline
\end{tabular}

depending on the affected site. ${ }^{9-11}$ Given the extensive lymphatic drainage in the supraglottis, the tumors on this site appear to spread earlier and to have a worse prognosis compared to other tumors on the other sites of the larynx, whereas in the glottis, tumors have a poorer lymphatic access and a lower chance of early spread. Tumors in the subglottis spread to areas such as the lymphatic vessels around the trachea, the inferior jugular vein, and the mediastinum via cricothyroid and cricotracheal lymphatics. In a cohort study conducted in Canada by MacNeil et al. on the survival of patients with laryngeal cancer, the mean survival rate was reported as about $54.4 \%$ when the glottis was involved and patients with subglottis tumor had a shorter longevity compared to those whose glottis was affected..$^{18}$ In the present study, subglottic tumors had the lowest survival rate; however, no significant differences were observed in the mean survival with respect to the location of the tumor in the different stages of the tumor. In a previous study of Canada, the number of patients admitted in early stages (stages I and II) of the disease was higher than the number of patients admitted in advanced stages (stages III and IV) and our study is not in line with their study. ${ }^{18}$ In a study published by the American Cancer Society, only $38.5 \%$ of the patients admitted to their center were in early stages (stages I and II) and 62.5\% were in advanced stages (stages III and IV). ${ }^{7}$ Also, in the present study, the number of patients admitted at advanced stages (stages III and IV) was higher compared with the ones admitted at early stages (stages I and II). In addition, the mean survival rate (irrespective of the prognostic factors) was much lower in stage IV than in the other stages, which is consistent with the findings of other studies, indicating that patients in stage IV appear to have a shorter chance of survival compared to patients in the other stages of laryngeal cancer. $6,7,7,18$

At its early stages, laryngeal squamous cell carcinoma is treated with surgery alone (laser surgery) or combined with radiotherapy, and the choice of treatment methods depends on the affected site and the decision to preserve the laryngeal function. Small tumors that have not spread to the lymph nodes have good prognoses and can have a recovery rate of $75-95 \%$ depending on the affected site and the degree of infiltration. ${ }^{19,21}$ Radiation or surgery can be used for treating these early-stage tumors (stages I and II), whereas total laryngectomy and postoperative chemoradiation appear to be appropriate methods for the treatment of tumors in advanced stages (stages III and IV). ${ }^{22-24}$ Today, the focus of treatment for tumors in advanced stages is to use a treatment protocol including chemoradiation without a total laryngectomy; however, according to previous published articles, the patients' survival rate seems to have reduced with this method of treatment (Gourin). ${ }^{25}$ Previous studies have examined and compared two treatment protocols for stages I and II of the disease, including surgery and/or radiotherapy, and showed no significant differences between them in terms of the patients' survival rate, although surgery was associated with a slightly higher mean survival rate compared to only radiotherapy. ${ }^{20,21}$ In the present study, patients in stages I and II who had not undergone laryngectomy showed a lower mean survival rate compared to those who had undergone a total or partial laryngectomy, although the difference was not statistically significant. Today, the treatment of patients in advanced stages involves the treatment protocol of preserving the larynx function, through chemoradiation for instance. ${ }^{26}$ Nevertheless, recent studies have shown that most patients who have used this treatment method have suffered late toxicity; as a result, it has been recommended to revise the decision to use this treatment and to seriously consider surgery, which is still a very effective treatment method, in case the tumors are large. In a study by Gourin et al. on survival rate in patients with advanced stage IV laryngeal cancer, the survival rate was found to be significantly higher in patients who had undergone surgery compared with those who had received only chemotherapy or a combination of chemotherapy and radiotherapy. ${ }^{25}$ In the present study, the best prognosis for patients in stage IV was observed in those who had received a combination of chemotherapy, radiotherapy, and surgery, followed by those who had received a combination of radiotherapy and surgery, and those who had not undergone surgery had a lower survival rate. In a study conducted by Lai et al. on patients in stage IV of laryngeal cancer, no significant difference was observed between the patients who had undergone surgery and those who had not. ${ }^{27}$ Based on the present study and the study conducted by Gourin et al., it appears that, even in stage IV of laryngeal cancer, better outcomes and higher survival rates can be expected with surgery. ${ }^{25}$

The important things to consider in the treatment of patients with laryngeal cancer include the quality of life and postoperative complications. Researchers have, therefore, come to believe that, in early stages (stages I and II), the best option is to use minor surgeries combined with radiation. In a study conducted by Lim et al. ${ }^{28}$ on the prognosis of patients in stages I and II of laryngeal cancer undergoing radiation, higher rates of recovery were observed in patients receiving this method of treatment (laser surgery combined with radiotherapy). Moreover, none of the patients in the present study had received only radiation without laryngectomy. Levy et al. conducted a study on the type of treatment and prognosis in patients in stages I and II of the disease and concluded that preserving the larynx and using one method of treatment alone or with radiation result in a good prognosis, which was inconsistent with the results of the present study. ${ }^{29}$ Harada et al. conducted a 
study on stages I and II of laryngeal cancer and their method of treatment and concluded that an initial radiation therapy together with the preservation of the larynx comprised the best treatment strategy. ${ }^{30}$ Stankovic et al. conducted a study on patients with advanced laryngeal cancer (stages III and IV) and observed that patients who had received radiotherapy or chemoradiation prior to laryngectomy had greater local complications compared to patients who had undergone surgery from the outset; however, they found that a 5-year survival depended on the stage of the tumor and its TNM status rather than the type of treatment. ${ }^{31}$

\section{Conclusion}

Overall, surgery and radiotherapy can be said to produce better survival rates in patients in stages I and II of laryngeal cancer compared to the other methods of treatment. In this stage, survival rate and the response to treatment are much better in younger patients compared with that in older ones. In patients in advanced stages (stages III and IV) of laryngeal cancer, a combination of surgery and radiotherapy or surgery and chemoradiation produces better survival rates and a greater response to treatment compared to when laryngectomy is not performed.

\section{Acknowledgment}

The authors thank all those who helped the writing this article.

\section{References}

1. Reiter R, Hoffmann TK, et al. Hoarseness-causes and treatments. Dtsch Ärztebl Int 2015;112(19):329-337. DOI: 10.3238/arztebl.2015.0329.

2. Schultz P. Vocal fold cancer. Eur Ann Otorhinolaryngol Head Neck Dis 2011;128(6):301-308. DOI: 10.1016/j.anorl.2011.04.004.

3. Mau T. Diagnostic evaluation and management of hoarseness. Med Clin North Am 2010;94(5):945-960. DOI: 10.1016/j.mcna.2010.05.010.

4. Luke CG, Yeoh E, et al. Exploring trends in laryngeal cancer incidence, mortality and survival: implications for research and cancer control. Asian Pac J Cancer Prev 2008;9(3):397-402.

5. Chatenoud L, Garavello W, et al. Laryngeal cancer mortality trends in European countries. Int J Cancer 2015;138(4):833-842.

6. Howlader N, Noone AM, et al. SEER Cancer Statistics Review, 1975-2011. Bethesda, MD: National Cancer Institute; April 2014. http:// seer.cancer.gov/csr/1975_2011/, based on November 2013 SEER data submission, posted to the SEER web site.

7. Howlader N, Noone AM, et al. SEER Cancer Statistics Factsheets: Larynx Cancer, 1975-2011. Bethesda, MD: National Cancer Institute; April 2014. http://seer.cancer.gov/csr/1975_2011/, based on November 2013 SEER data submission, posted to the SEER web site.

8. Kolahdoozan S, et al. Five common cancers in Iran. Arch Iran Med 2010;13(2):143-146.

9. Mafi N, Kadivar M, et al. Head and neck squamous cell carcinoma in Iranian patients and risk factors in young adults: a fifteen-year study. Asian Pac J Cancer Prev 2012;13(7):3373-3378. DOI: 10.7314/ APJCP.2012.13.7.3373.

10. Saedi B, Razmpa E, et al. The epidemiology of laryngeal cancer in a country on the esophageal cancer belt. Indian J Otolaryngol 2009;61(3):213-217. DOI: 10.1007/s12070-009-0069-6.

11. Risks and causes of laryngeal cancer, Cancer Research UK: http://www. cancerresearchuk.org/about-cancer/type/larynx-cancer/about/risksand causes-of-laryngeal-cancer, May 26, 2015.

12. Grenman R, Koivunen P, et al. Laryngeal cancer in Finland. Duodecim 2015;131(4):331-337.
13. Al-Mamgani A, Tans $L$, et al. A single-institutional experience of 15 years of treating T3 laryngeal cancer with primary radiotherapy, with or without chemotherapy. Int J Radiat Oncol Biol Phys 2012;83(3):1000-1006. DOI: 10.1016/j.ijrobp.2011.07.045.

14. Chen AY, Fedewa $S$, et al. Improved survival is associated with treatment at high-volume teaching facilities for patients with advanced stage laryngeal cancer. Cancer 2010;116(20):4744-4752. DOI: 10.1002/cncr.25364.

15. Doornaert $\mathrm{P}$, Terhaard CH, et al. Dutch National Platform Radiotherapy $\mathrm{H}$, Neck C. Treatment of T3 laryngeal cancer in the Netherlands: a national survey. Radiat Oncol 2015;10:134. DOI: 10.1186/s13014-0150440-6.

16. Timmermans AJ, de Gooijer CJ, et al. T3-T4 laryngeal cancer in The Netherlands Cancer Institute; 10-year results of the consistent application of an organ-preserving/-sacrificing protocol. Head Neck 2015;37(10):1495-1503. DOI: 10.1002/hed.23789.

17. Dziegielewski PT, O'Connell DA, et al. Primary total laryngectomy versus organ preservation for T3/T4a laryngeal cancer: a populationbased analysis of survival. J Otolaryngol 2012;41(Suppl 1): S56-S64.

18. MacNeil SD, Liu K, et al. Secular trends in the survival of patients with laryngeal carcinoma, 1995-2007. Curr Oncol 2015;22(2):e85-e99. DOI: 10.3747/co.22.2361.

19. National Comprehensive Cancer Network, Inc. 2014, Available at http://www.nccn.org. May 12, 2013.

20. Warner L, Chudasama J, et al. Radiotherapy versus open surgery versus endolaryngeal surgery (with or without laser) for early laryngeal squamous cell cancer. Cochrane Database Syst Rev 2014;12:CD002027. DOI: 10.1002/14651858.CD002027.pub2.

21. Qin J, Wang H, et al. Small partial laryngectomy without tracheotomy for T1-2 stage glottic carcinoma. Lin Chung Er Bi Yan Hou Tou Jing Wai Ke Za Zhi 2014;28(16):1272-1274.

22. Forastiere AA, Goepfert $\mathrm{H}$, et al. Concurrent chemotherapy and radiotherapy for organ preservation in advanced laryngeal cancer. N Engl J Med 2003;349(22):2091-2098. DOI: 10.1056/NEJMoa031317.

23. Ganly I, Patel SG, et al. Predictors of outcome for advanced-stage supraglottic laryngeal cancer. Head Neck 2009;31(11):1489-1495.

24. Caicedo-Granados E, Beswick DM, et al. Oncologic and functional outcomes of partial laryngeal surgery for intermediate-stage laryngeal cancer. Otolaryngol Head Neck Surg 2013;148(2):235-242. DOI: 10.1177/0194599812466367.

25. Gourin CG, Conger BT, et al. The effect of treatment on survival in patients with advanced laryngeal carcinoma. The Laryngoscope 2009;119(7):1312-1317. DOI: 10.1002/lary.20477.

26. Rosenthal DI, Mohamed AS, et al. Long-term outcomes after surgical or nonsurgical initial therapy for patients with T4 squamous cell carcinoma of the larynx: A 3-decade survey. Cancer 2015;121(10): 1608-1619. DOI: 10.1002/cncr.29241.

27. Lai FY, Zhang Q, et al. Treatment and prognosis of stage IV glottic laryngeal cancer. Chinese J Cancer Res 2008;27(1):71-74.

28. Lim YJ, Wu HG, et al. Long-Term Outcome of Definitive Radiotherapy for Early Glottic Cancer: Prognostic Factors and Patterns of Local Failure. Cancer Res Treat 2015;47(4):862-870. DOI: 10.4143/crt.2014. 203.

29. Levy A, Blanchard $P$, et al. Squamous cell carcinoma of the laryn $x$ with subglottic extension: is larynx preservation possible? Strahlenther Onkol 2014;190(7):654-660. DOI: 10.1007/s00066-014-0647-8.

30. Harada A, Sasaki R, et al. Treatment outcomes of the patients with early glottic cancer treated with initial radiotherapy and salvaged by conservative surgery. Jpn J Clin Oncol 2015;45(3):248-255. DOI: 10.1093/jjco/hyu203.

31. Stankovic M, Milisavljevic $D$, et al. Primary and salvage total laryngectomy. Influential factors, complications, and survival. J BUON 2015;20(2):527-539. 